\title{
Mathematical modeling in diabetic retinopathy
}

\author{
I $V$ Vorobyeva ${ }^{1, *}$ \\ ${ }^{1}$ Russian Medical Academy of Continuous Professional Education, 2/1, Barrikadnaya Street, \\ Moscow, 125993, Russia.
}

\begin{abstract}
The problem of digital medicine in ophthalmology arose in connection with the increase in the incidence of diabetes mellitus in the world. The issue of preventing blindness and early diagnosis in diabetic retinopathy with a forecast of its course using mathematical modeling is especially acute. A system for assessing the prognosis of the risk of developing diabetic retinopathy was created based on interdisciplinary research in three areas: ophthalmology, biochemistry and mathematics. The studies were conducted in ophthalmology with the analysis of the condition of the retina in the macular area using digital ophthalmic equipment (optical coherence tomograph, fundus microperimeter). Biochemical studies consisted in the analysis of tear fluid with the determination of the level of the pathogenetic biomarker VEGF-A and the analysis of blood serum with the determination of the level of glycated hemoglobin $\mathrm{HbAl}$. Mathematical research consisted in the presentation of digital material in the form of mathematical criteria based on the method of standardizing the indicator under study. The result of the research was a computer program based on the developed mathematical formulas. The program gives the opportunity to automatically enter the data of a specific patient with diabetic retinopathy and give a prognosis of the disease.
\end{abstract}

\section{Introduction}

\subsection{The problem of blindness in diabetic retinopathy}

Blindness in diabetic retinopathy occurs 25 times more often than in other diseases. Diabetic macular edema gives a particularly difficult prognosis for vision. Currently, the incidence of diabetes mellitus in the world is steadily growing so much that they talk about a pandemic. For the prevention of blindness and low vision, the issues of early diagnosis of the disease are especially relevant $[1,2]$.

\subsection{Early diagnosis issues using a computer, tomograph and perimeter}

Early diagnosis of diabetic retinopathy became possible with the advent of medical devices such as an optical coherence tomograph [3-8] and a fundus microperimeter $[9,10]$. Interdisciplinary research plays an important role in the diagnosis of diseases. Especially

\footnotetext{
Corresponding author: irina.docent2000@mail.ru
} 
important is the identification of pathogenetic biomarkers in the lacrimal fluid and blood serum [11-13]. The leading position is occupied by interdisciplinary research on mathematical modelling in various fields of science [14,15] and programming [16-18], including medicine area $[19,20]$.

\subsection{The problem of implementing mathematical modeling and programming in diabetic retinopathy}

The use of methods of mathematical modeling, programming, digital technologies in medicine [21] and ophthalmology [22] is crucial. This is related to the need to simplify the analysis of a large amount of digital material for systematizing data from an optical coherence tomograph and a microperimeter in diabetic retinopathy [23]. Mathematical models and digital technologies in medicine should take into account not only digital data of medical equipment, but also the individual characteristics of the course of the underlying disease in a patient with diabetes mellitus according to the levels of glycated hemoglobin and pathogenetic biomarkers in the lacrimal fluid. This requires interdisciplinary research: ophthalmological, biochemical, mathematical with the creation of computer programs [24,25].

\section{Problem}

To develop a mathematical model and a computer program for predicting the development of diabetic retinopathy based on a comprehensive analysis of digital data from ophthalmic equipment and the levels of biomarkers in the lacrimal fluid and blood serum.

\section{Materials and methods}

\subsection{Methods of examination of patients using ophthalmic equipment and analysis of laboratory data of biomarkers in tear and blood serum}

All patients underwent determination of the maximum corrected visual acuity, intraocular pressure, as well as examination of the anterior segments of the eye, optical coherence tomography, fundus microperimetry, the level of vascular endothelial growth factor VEGF$\mathrm{A}$ in the lacrimal fluid and the level of glycated hemoglobin HbAlc in the blood serum.

Optical coherence tomography was performed on an RTVue-100 tomograph (OptoVue, the USA). Optical coherence tomography (OCT) is based on the principle of low-coherence interferometry. Imaging is based on comparing the time difference between the beam reflected at different depths from the retinal structures and the reference beam coming from the oscillating mirror. The A-scan displays the intensity of the reflected beam from various retinal structures at a single scan point. The set of many A-scans gives the opportunity to get a B-image. Scanning speed: 26,000 A-scans per second. Scanning depth: up to $2.3 \mathrm{~mm}$. Scan length $2 \mathrm{~mm}-12 \mathrm{~mm}$. Diopter compensation range: $-15 \mathrm{D} /+20 \mathrm{D}$. A-scan resolution up to 1024 pixels. B-scan resolution up to 16384 A-scans.

Fundus microperimetry was performed using the MAIA perimeter (CenterVue Spa, Italy). MAYA includes: a linear scanning laser ophthalmoscope, a high-frequency eye tracker, an automated macular perimeter, software, a regulatory framework and a statistical 
analysis module. The device is controlled during the research and processing the results using a touch screen and two buttons on the front panel.

The level of VEGF-A in the SG was assessed using an enzyme-linked immunosorbent assay using the Quantikine ELISA VEGF Immunoassay kit Six Pack (R\&D Systems, USA) on a Perkin-Elmer Victor X3 luminometer (Perkin-Elmer, USA).

The level of glycated hemoglobin and glucose was assessed by the standard method.

Fundus photographic recording was conducted using an NM-1000 color fundus camera (Nidek, Germany).

\subsection{Development of a decision support system for a doctor using a mathematical model for the development of digital medicine methods}

To work with the presented large array of digital data of optical equipment and laboratory studies of biomarker levels, a system of criteria that allows for a quantitative assessment of the patient's condition was developed.

To assess the prognosis of diabetic retinopathy with diabetic macular edema, it was proposed to use the following evaluation criteria: the thickness of the macular zone in 9 areas using an optical coherence tomograph, the intensity of light stimuli of the retina at 37 points using an optical coherence tomograph, the level of glycated hemoglobin HbA1c in the blood serum and the level vascular endothelial growth factor VEGF-A in the lacrimal fluid.

A system of criteria that describes the prognosis of the development of diabetic retinopathy in patients with type 2 diabetes mellitus is presented:

$$
\left\{\begin{array}{l}
R_{1}=\frac{\sum_{1}^{9}\left(\mathrm{~h}_{i}-\mathrm{h}_{\text {min }}\right)}{\left(\mathrm{h}_{\text {max }}-\mathrm{h}_{\text {min }}\right) \cdot \mathrm{m}} \\
R_{2}=\frac{\sum_{1}^{36}\left(I_{i}-I_{\text {min }}\right)}{\left(I_{\text {max }}-I_{\text {min }}\right) \cdot 36} \\
R_{3}=\frac{G}{G_{c r i}} \\
R_{4}=\frac{V E G F}{V E G F_{\text {max }}} \\
R_{\text {general }}=R_{1} \cdot R_{2} \cdot R_{3} \cdot R_{4}
\end{array} .\right.
$$

where $R_{1}$ - retinal thickness criterion;

$R_{2}$ - sensitivity criterion;

$R_{3}$ - glycated hemoglobin effect criterion; 
$R_{4}$ - criterion of the influence of the vascular endothelial growth factor level;

$R_{\text {general }}$ - integral criterion of progression of the disease;

$\mathrm{h}_{i}$ - thickness of retinal sectors (in each sector of the macular zone), $\mu m$;

$I_{i}$ - light stimulus intensity, $d b$;

$\mathrm{h}_{\text {min }}$ - retinal thickness in one of the scanning sectors of the macular zone, excluding the central and extreme sectors, $\mu m$;

$I_{\text {min }}-$ minimum light stimulus intensity, $d b$;

$\mathrm{h}_{\max }-$ maximum retinal thickness of the macular zone, $\mu \mathrm{m}$;

$I_{\text {max }}$ - maximum light stimulus intensity, $d b$;

36 - the number of points at which the light stimuli are determined;

$\mathrm{m}$ - number of retinal scan sectors $(\mathrm{m}=9)$;

$G$ - glycated hemoglobin level from the studied patient, \%;

$V E G F$ - level of vascular endothelial growth factor in the lacrimal fluid from the studied patient, $p g / m l$;

$G_{c r i}$ - upper limit of glycated hemoglobin level, \%;

$V E G F_{\text {max }}$ - maximum level of endothelial growth factor, $\mathrm{pg} / \mathrm{ml}$.

The boundary value table for the prognosis of diseases (table 1) was developed on the basis of statistical data processing. 
Table 1. The prognosis of the disease depending on the criterion $R_{\text {general }}$.

\begin{tabular}{|c|c|c|}
\hline$R_{\text {general }}$ & Disease diagnosis & Disease prognosis \\
\hline$R_{\text {general }} \leq 0.07$ & $\begin{array}{c}\text { Non-proliferative stage } \\
\text { of diabetic retinopathy } \\
\text { with diabetic macular } \\
\text { edema }\end{array}$ & $\begin{array}{c}\text { Low risk of disease } \\
\text { progression with a good } \\
\text { prognosis for vision }\end{array}$ \\
\hline $0.07<R_{\text {general }}<0.18$ & $\begin{array}{c}\text { Preproliferative stage of } \\
\text { diabetic retinopathy with } \\
\text { diabetic macular edema }\end{array}$ & $\begin{array}{c}\text { High risk of disease } \\
\text { progression with a bad } \\
\text { prognosis for vision }\end{array}$ \\
\hline$R_{\text {general }} \geq 0.18$ & $\begin{array}{c}\text { Proliferative stage of } \\
\text { diabetic retinopathy with } \\
\text { diabetic macular edema }\end{array}$ & $\begin{array}{c}\text { High risk of disease } \\
\text { progression with a bad } \\
\text { prognosis for vision }\end{array}$ \\
\hline
\end{tabular}

\section{Results and discussion}

\subsection{Algorithm of a computer program}

Based on digital data of ophthalmic equipment, laboratory study of biomarker levels in tear fluid and blood serum, and the proposed criteria a computer program that automatically determines the prognosis of the disease was developed. The program consists of a data input module, a calculation module and a data output module.

The data entry module is used to enter data from the optical coherence tomograph, the MAIA fundus microperimeter and laboratory examination of the patient's biomarker levels.

The program enables the doctor to enter data from the optical coherence tomograph in the form of retinal thicknesses in 9 sectors of the macular zone and data on the presence or absence of edema in each sector of the macular zone. Data entry from the MAIA fundus microperimeter in the form of inputting the number of points with a certain light stimulus was implemented (it is necessary to enter data on the intensity at 37 points of the macular zone). The last data of the patient's laboratory examination is entered into the program in the form of the level of glycated hemoglobin in the blood serum HbAlc and the level of the vascular endothelial growth factor VEGF-A in the lacrimal fluid. For the convenience of an ophthalmologist, the interface of the program repeats the interfaces of ophthalmological equipment in its color scale.

The calculation module implements algorithms for logical operations and mathematical operations. Logical operations are an exception from the calculation of the retinal thickness criterion $R_{1}$ of areas of the macular zone where there is retinal edema, checking the correctness of the input of the macular photosensitivity according to the histogram of the fundus microperimeter MAIA, comparing the levels of biomarkers with their limit values. The algorithm of mathematical operations is a sequential calculation of the retinal thickness criterion $R_{1}$, sensitivity criterion $R_{2}$, glycated hemoglobin effect criterion $R_{3}$, criterion of the influence of the vascular endothelial growth factor level $R_{4}$, integral criterion of 
progression of the disease $R_{\text {general }}$ (system of equations (1)). After the calculation, a logical operation is performed to compare the obtained value of the criterion with the prognosis of the disease according to table (1). The data output module displays data on the prognosis of the disease on the computer screen as a text message. The algorithm of the program is shown in Figure 1.

\subsection{Clinical example of a patient}

The work of the program is demonstrated on a specific clinical example of the patient who underwent examination: traditional ophthalmological examination, including photo registration of the fundus, optical coherence tomography, fundus microperimetry MAIA, biomarkers in the lacrimal fluid and blood serum.

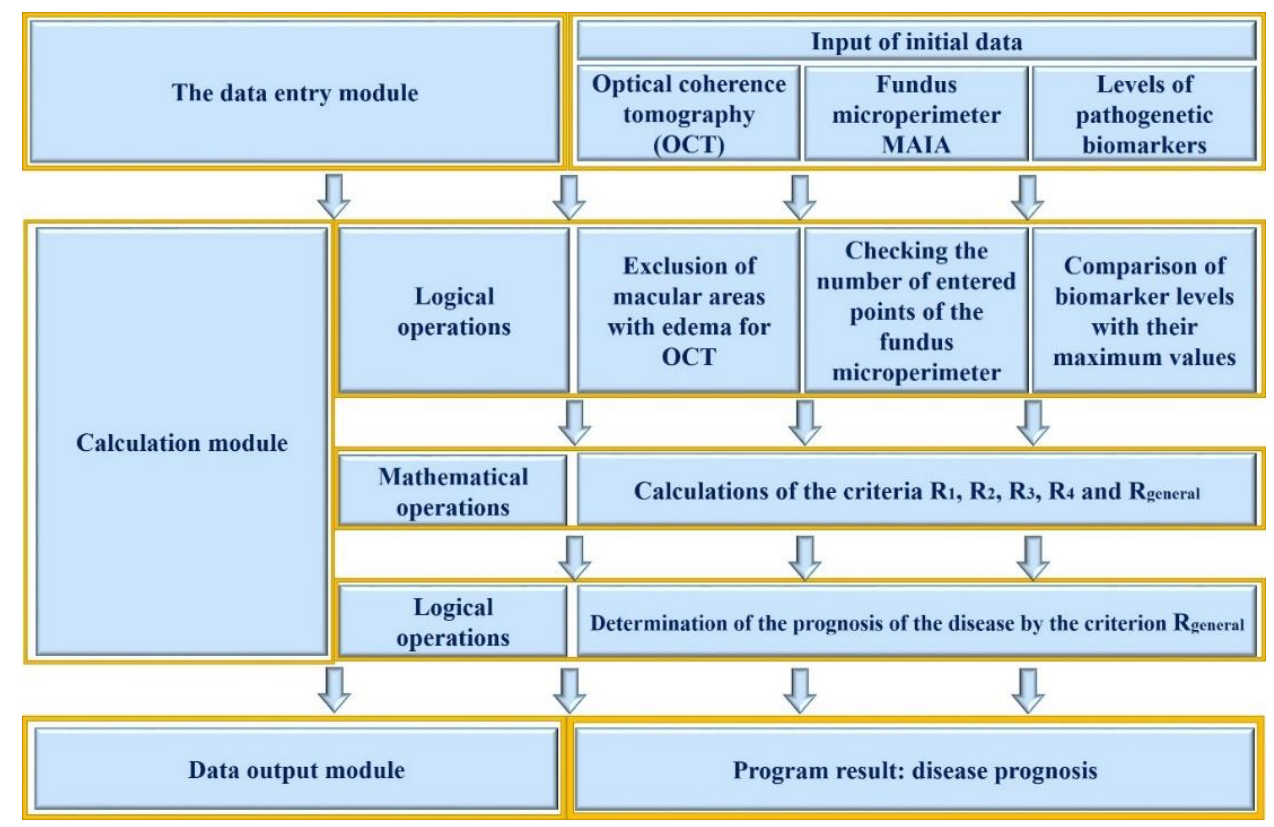

Fig. 1. Algorithm of the program.

Patient K., 63 years old, has been suffering from type 2 diabetes mellitus (non-insulin dependent) for 10 years, the $\mathrm{HbA} 1 \mathrm{c}$ level is $7.2 \%$, the level of vascular endothelial growth factor VEGF-A in the lacrimal fluid is $800 \mathrm{pg} / \mathrm{ml}$.

Vision is reduced to 0.2 in both eyes. Intraocular pressure is normal and in the lenses there are initial opacities under the posterior capsule. In the vitreous body there are multiple floating opacities. On the fundus the optic disc is pale pink, the boundaries are clear, the veins are full-blooded, the arteries are narrowed. In the macular zone multiple foci of white color are revealed, on the periphery of the retina there are single punctate hemorrhages.

The state of the fundus is illustrated according to the results of objective photographic registration (figure 2 and figure 3 ). 
For the considered clinical case of the patient, data from digital ophthalmic equipment and biochemical markers was entered into the program. The calculation in the program was conducted according to the presented data of patient's left eye.

The input boxes for entering data into the computer program for the fundamental microperimeter MAIA (figure 4 and figure 5) are presented.

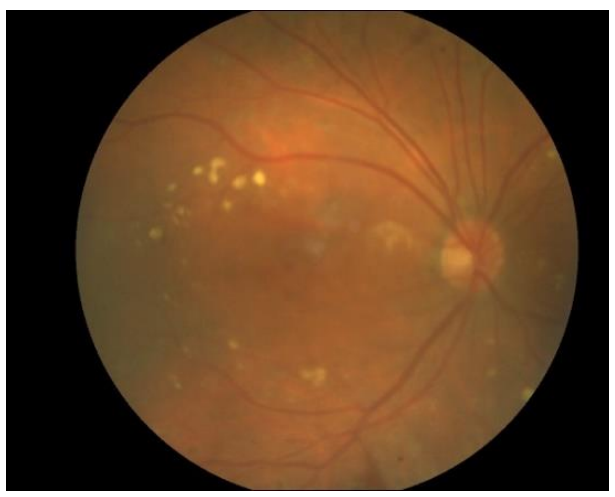

Fig. 2. Photo registration of the right eye.

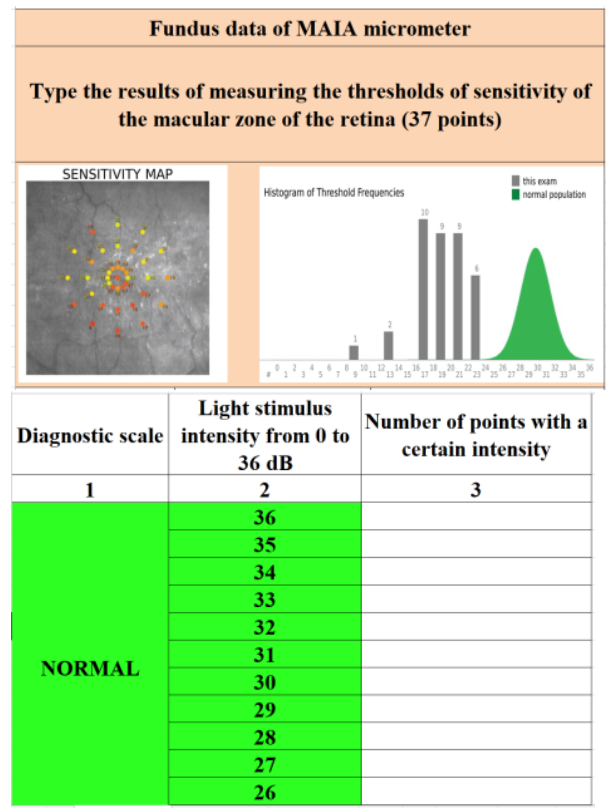

Fig. 4. Data Entry for Fundus Microperimeter MAIA (Part 1).

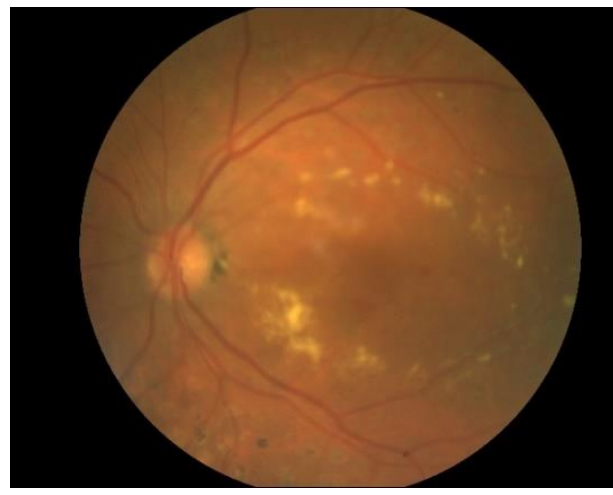

Fig. 3. Photo registration of the left eye.

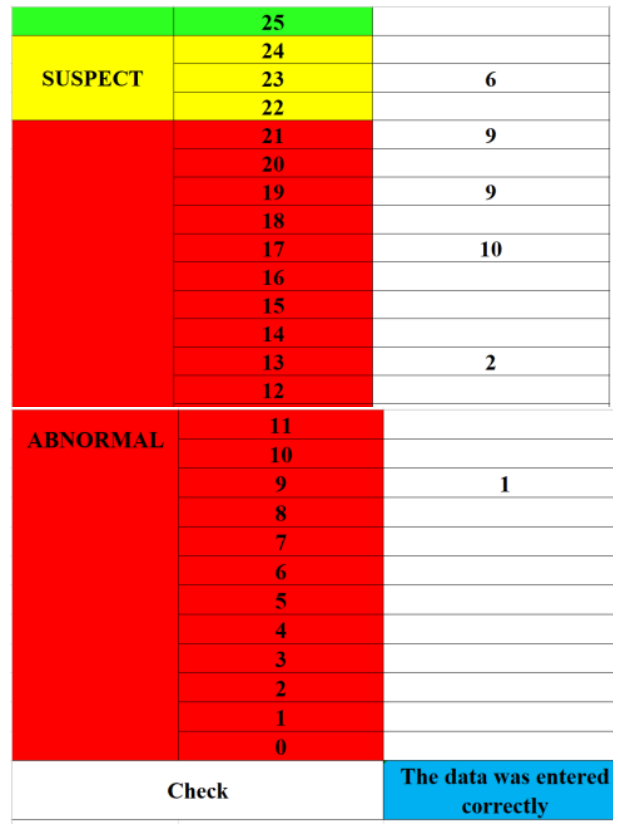

Fig. 5. Data entry for the MAIA fundus microperimeter (part 2).

The result of the program is the formulation of the prognosis of the disease. 
This individual patient prognosis is "low risk of disease progression with a good prognosis for vision". The calculation results of the program coincide with the doctor's clinical data.

The effectiveness of the program for the prognosis of the disease is determined by the use of a comprehensive analysis of digital data of ophthalmic equipment and pathogenetic biomarkers in the lacrimal fluid and blood serum of a particular patient according to a mathematical model. This complex interdisciplinary approach enables the opghalmologist to approach a personalized and digital medicine in the prognosis of eye diseases, which is more effective compared to individual diagnostic methods. The input boxes for entering data into a computer program for an optical coherence tomograph (figure 6) and laboratory data of biomarker levels (figure 7) are presented.

An input box for entering data of a computer program with a disease prognosis is presented (figure 8).

\section{Conclusion}

The levels of pathogenetic biomarkers in the lacrimal fluid and blood serum were analyzed. Also, the clinical and morphofunctional features of the retina in diabetic retinopathy and diabetic macular edema at different stages of the disease were studied. The obtained results made it possible to develop a system of criteria for assessing the thickness of the retina in the macular zone, determining the photosensitivity of the macula, and the levels of pathogenetic biomarkers.

\begin{tabular}{|c|c|c|c|c|}
\hline \multicolumn{5}{|c|}{ Tomograph data } \\
\hline \multicolumn{5}{|c|}{ Type the results of measuring retinal thickness } \\
\hline № & Retinal zones & $\begin{array}{c}\text { Macular area } \\
\text { scanning sector }\end{array}$ & $\begin{array}{c}\text { Retinal } \\
\text { thickness, } \\
\text { microns }\end{array}$ & Edema (yes/no) \\
\hline 1 & 2 & 3 & 4 & 5 \\
\hline 1 & $\begin{array}{c}\text { Fovea } \\
\text { centralis }\end{array}$ & Centre & 294 & yes \\
\hline 2 & \multirow{4}{*}{ ParaFovea } & Tempo & 423 & yes \\
\hline 3 & & Superior & 389 & no \\
\hline 4 & & Nasal & 356 & no \\
\hline 5 & & Inferior & 332 & yes \\
\hline 6 & \multirow{4}{*}{ PeriFovea } & Tempo & 392 & yes \\
\hline 7 & & Superior & 320 & no \\
\hline 8 & & Nasal & 306 & no \\
\hline 9 & & Inferior & 283 & yes \\
\hline
\end{tabular}

Fig. 6. Data entry for an optical coherence tomograph.

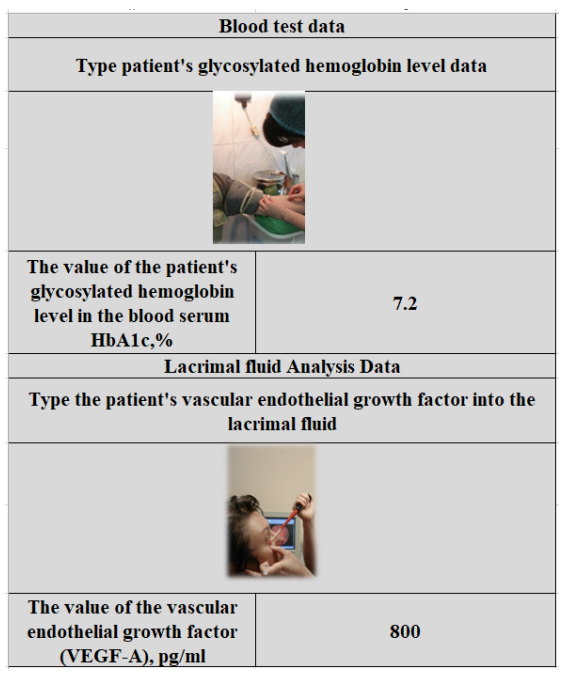

Fig. 7. Entering data on the levels of biomarkers HbA1c and VEGF-A.

\section{THE RESULT OF PROGRAM CALCULATIONS:}

\section{LOW RISK OF DISEASE PROGRESSION WITH A GOOD PROGNOSIS FOR VISION}

Fig. 8. Data entry box of the computer program with the formulation of the prognosis of the disease. 
A mathematical model has been created for a practical ophthalmologist. The model was obtained based on the analysis of digital data of medical ophthalmic equipment (optical coherence tomograph and perimeter) and data of pathogenetic biomarkers in the lacrimal fluid and blood serum. The created model gives the opportunity to predict the course of diabetic retinopathy.

A computer program that enables the doctor to enter the data of a particular patient (tomograph, perimeter, level of glycated hemoglobin, vascular endothelial growth factor) within 3-5 minutes and to predict diabetic retinopathy in an automatic mode has been developed.

The practical application of this program is widely implemented in the work of practical ophthalmologists in Russia in ophthalmological hospitals. This approach makes it possible to systematize the data of diabetic retinopathy (by the level of the vascular endothelial growth factor VEGF-A biomarker in the lacrimal fluid and glycated hemoglobin HbAlc in the blood serum and the clinical and morphofunctional state of the retina at different stages of the disease). The obtained prognosis of the disease according to the developed program allows the doctor to choose the correct algorithm for the pathogenetic approach to treatment.

Thus, digital medicine allows for a personalized approach to each patient and gives the opportunity to support the doctor's decision-making.

\section{References}

1. Striglia E, Caccioppo A, Castellino N, Reibaldi M and Porta M 2020 Expert Opinion on Emerging Drugs

2. Youngblood H, Robinson R, Sharma A and Sharma S Journal of Molecular Sciences 20(19) 4755

3. Kong R, Wu W, Qiu R, Gao L, Du F, Liu A, Cai X and Dai C 2020 Experimental Biology and Medicine 1535370220949834

4. Porwal S, Nithyanandam S, Joseph M and Vasnaik A K 2020 Indian Journal of Ophthalmology 68(8) 1584-6

5. Pinilla I, Idoipe M, Perdices L, Sanchez-Cano A, Acha J, Lopez-Galvez M I, Cuenca N, Abecia E and Orduna-Hospital E 2020 Journal of Retinal and Vitreous Diseases 40(7) 1379

6. Faghihi H, Riazi-Esfahani H, Khodabande A, Pour E K, Mirshahi A, Ghassemi F, Mirshahi R, Khojasteh H, Bazvand F, Hashemi A, Tayebi F, Faghihi S and Esfahani M 2020 European Journal of Ophthalmology 1120672120952642

7. Ganjee R, Moghaddam M E and Nourinia, R 2020 Images Medical Physics

8. Park, Y G, Kim M and Roh Y J 2020 Journal of Diabetes Research 6210865

9. Sharanjeet-Kaur, Ismail S A, Mutalib H A and Ngah N F 2019 Journal of Optometry 12(3) 174

10. Michalska A, Dorecka M, Jackiewicz K, Miniewicz-Kurkowska J, Sobieraj R, Michalski M and Romaniuk W 2013 Polskie Archiwum Medycyny Wewnetrznej-Polish Archives of Internal Medicine 123(3) 98

11. Vorobyeva I V, Moshetova L K and Shcherbakova E V 2017 Oftalmologiya 14(1) 40

12. Vorobyeva I V 2017 Oftalmologiya 14(1) 67

13. Vorobyeva I V 2018 Oftalmologiya 15(2) 189 
14. Zubarev K P and Gagarin V G 2021 Advances in Intelligent Systems and Computing (International Scientific Conference Energy Management of Municipal Facilities and Sustainable Energy Technologies EMMFT 2019) 1259593

15. Zubarev K P and Gagarin V G 2018 IOP Conference Series: Materials Science and Engineering (International Multi-Conference on Industrial Engineering and Modern technologies) 463032082

16. Gagarin V G, Akhmetov V K and Zubarev K P 2020 IOP Conference Series: Materials Science and Engineering (International Science and Technology Conference "FarEastCon-2019") 753022045

17. Gagarin V G, Akhmetov V K and Zubarev K P 2018 MATEC Web of Conferences (SPbWOSCE-2017) 17003014

18. Gagarin V G, Akhmetov V K and Zubarev K P 2020 IOP Conference Series: Materials Science and Engineering (International Science and Technology Conference "FarEastCon-2019") 753 L 022046

19. Saman G, Gohar N, Noor S, Shahnaz A, Idress S, Jehan N, Rashid R and Khattak S S 2020 Automatic detection and severity classification of diabetic retinopathy Multimedia tools and applications

20. Vaishnavi J, Ravi S and Anbarasi A 2020 Multimedia tools and applications.

21. Li F, Liu Z, Chen H, Jiang M, Zhang X and Wu Z 2019 Translational Vision Science and Technology 8(4) 4

22. Reddy S 2019 International Journal of Technology and Human Interaction 15(4) 30

23. Abbas Q, Fondon I, Sarmiento A, Jimenez S and Alemany P 2017 Medical and Biological Engineering and Computing 55(11) 1959

24. Roychowdhury S, Koozekanani D D and Parhi K K 2014 IEEE Journal of Biomedical and Health Informatics 18(5) 1717

25. Siddalingaswamy P C and Prabhu K G 2009 IFMBE Proceedings (13th International Conference on Biomedical Engineering) 23277 